\title{
Microcystin-LR detection in water by the Fabry-Pérot interferometer using an optical fibre coated with a sol-gel imprinted sensing membrane
}

\author{
Raquel B. Queirós, S.O. Silva, J.P. Noronha, O. Frazão, P. Jorge, G. Aguilar, \\ P.V.S. Marques, M.G.F. Sales
}

\begin{abstract}
A B S T R A C T
Cyanobacteria deteriorate the water quality and are responsible for emerging outbreaks and epidemics causing harmful diseases in Humans and animals because of their toxins. Microcystin-LR (MCT) is one of the most relevant cyanotoxin, being the most widely studied hepatotoxin. For safety purposes, the World Health Organization recommends a maximum value of $1 \mu \mathrm{g} \mathrm{L}^{-1}$ of MCT in drinking water. Therefore, there is a great demand for remote and real-time sensing techniques to detect and quantify MCT.

In this work a Fabry-Pérot sensing probe based on an optical fibre tip coated with a MCT selective thin film is presented. The membranes were developed by imprinting MCT in a sol-gel matrix that was applied over the tip of the fibre by dip coating. The imprinting effect was obtained by curing the sol-gel membrane, prepared with (3-aminopropyl) trimethoxysilane (APTMS), diphenyl-dimethoxysilane (DPDMS), tetraethoxysilane (TEOS), in the presence of MCT. The imprinting effect was tested by preparing a similar membrane without template.

In general, the fibre Fabry-Pérot with a Molecular Imprinted Polymer (MIP) sensor showed low thermal effect, thus avoiding the need of temperature control in field applications. It presented a linear response to

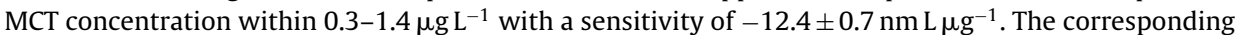
Non-Imprinted Polymer (NIP) displayed linear behaviour for the same MCT concentration range, but with much less sensitivity, of $-5.9 \pm 0.2 \mathrm{~nm} \mathrm{~L} \mathrm{\mu g}^{-1}$. The method shows excellent selectivity for MCT against other species co-existing with the analyte in environmental waters. It was successfully applied to the determination of MCT in contaminated samples. The main advantages of the proposed optical sensor include high sensitivity and specificity, low-cost, robustness, easy preparation and preservation.
\end{abstract}

Keywords:

Microcystin-LR

Molecular imprinting

Sol-gel

Fabry-Pérot interferometer

Freshwater

\section{Introduction}

Some species of Cyanobacteria may release, to water bodies, several toxins, when their cells rupture or die. The most common of the cyanobacterial toxins found in water are microcystins, particularly MCT. Microcystins are very stable in water, keeping their chemical structure in warm/cold water and against radical changes in $\mathrm{pH}$. Humans are most likely to be exposed to microcystins through the consumption of contaminated drinking water or recreational activities such as swimming (Kuiper-Goodman et al., 1999; Swanson and Hammer, 2000; Vivegnis et al., 1999). In general, these toxins cause skin allergy, attack the liver (hepatotoxins) or the nervous system (neurotoxins), playing a potent and dreadful role on living beings (Carmichael, 1992; WHO, 1998). They are also the ones most often responsible for poisoning animals and humans who come into contact with toxic blooms. Due to their environmental persistence, widespread distribution, high toxicity, and threat to public health, the levels of Microcystins have become an important parameter in water quality control, environmental monitoring, and toxicology. The World Health Organization (WHO) has recommended a maximum value of $1 \mu \mathrm{g} \mathrm{L}^{-1}$ for microcystins in drinking water (WHO, 1998).

The extremely low recommended values of MCT in water are reached only by few techniques (Sangolkar et al., 2006). Only liquid chromatography (Neffling et al., 2010), ELISA (Long et al., 2009b), capillary electrophoresis (Birungi and Li, 2009), luminescence (as biosensing technology) (Jasionek et al., 2010; Long et al., 2008, 2009a,c) or a combination the previous ones (Triantis et al., 2010) have been used for this purpose. In essence, all of them employed immuno- or enzymatic assays. These are highly selective because 
they rely of lock/key based interactions, just as those of antibody/antigen nature. However, as they rely on biomaterials with short life-time and specific requisites for storage, they turn out expensive and require specific handling procedures. Furthermore, some of the previous methods cannot operate in locus, as required in crisis management when some public facility is suspected of contamination or simply for routine pollution monitoring. They may also be time-consuming and require expensive instrumentation and special reagents (Wang et al., 2009).

The search for alternative procedures for real-time bacteria control requires the development of methods that combine sensitive and selective recognition capabilities of low quantities of analyte and sensory systems with rapid response and long term stability. A high sensitivity is here fundamental since one must often detect only a few molecules (Li et al., 2008). A suitable optical biosensor design could meet these criteria by employing an optical fibre interferometer (Jesus et al., 2009; Silva et al., 2008). For this purpose, the optical fibre tip must be covered with an element of recognition that enables a selective MCT detection. This may be achieved by coating the fibre with a compatible sensing material such as sol-gel with MCT imprinted.

Optical fibre interferometers are particularly suitable when very low concentrations should be detected in a regular fashion. An interferometer combines two or more light waves in an optical instrument in such a way that interference takes place between them. In fibre optic interferometer Fabry-Pérot (FFPI) the interference occurs at the partially reflecting end face surface of the fibre and an external mirror. The size of the sensitive element based on this principle can be as small as the diameter of the fibre (about $0.1 \mathrm{~mm}$ ), presenting a high signal to noise ratio. Obviously, the selectivity here is attributed to the sensing membrane covering the optical fibre.

Sol-gel is transparent in the visible region of the spectra, thus being a compatible material with an optical fibre and interferometric measures. Sol-gel is a well-known process concerning transition of a system from liquid 'sol' (the colloidal suspension of particles) into solid 'gel' (Mac-Craith et al., 1997). The chemistry of sol-gel processes includes the hydrolysis of a precursor, condensation of the hydrolysis product to form a sol solution, gelatinizing the sol solution to form a gel, drying the formed gel and heat treatment of the dried sol-gel material (Dunn and Zink, 1991; Mujahid et al., 2010). Generally, metal alkoxides are used as precursors because they hydrolyze when they are exposed to water. The partially hydrolyzed alkoxide molecules react with each other or with the un-hydrolyzed ones to undergo a condensation reaction and form a cross-linked matrix liberating water or alcohol (Mujahid et al., 2010). In the end, an interconnected, homogenous and rigid material is obtained. Sol-gel films offer great potential on the development of optical sensors due to their transparency in the visible region (Dunn and Zink, 1991; Lev, 1992). The selectivity of this material to a specific compound may be attributed by molecular imprinting (MI) technology.

$\mathrm{MI}$ is the imprint of a certain molecule in a rigid polymeric matrix. The template molecule is removed without disturbing the geometry of the solid matrix. The molecular imprinted polymer (MIP) keeps the ability to rebind the template because of its 3-D functional arrangement (Mosbach, 1994). Template rebind produces dielectric changes in the nanostructure sensor, thus enabling its detection (Díaz-García and Laíno, 2005).

In this work, a FFPI sensing device with a MI sol-gel membrane at the cleaved end of an optical fibre is presented for detection of MCT. All the results were compared with those of a non-imprinted polymer (NIP) obtained by removing the template from the sol-gel preparation.

\section{Theory and setup of the interferometer}

Optical interferometry combines more than one light wave and allows them to interfere with each other. For thin films with thickness $d$, the incident light at a specific wavelength $\lambda$ constructively interferes when Eq. (1) is satisfied, where $m$ is the order of interference, $n$ is the refractive index of the film and $\theta_{\mathrm{t}}$ is the angle of the transmitted light.

$m \lambda=2 n d \cos \theta_{\mathrm{t}}$

The Fabry-Pérot interferometer makes use of multiple beam reflections which follow the interference condition for thin films. It consists of two identical mirrors separated by a uniform cavity with length $L$, as shown in Fig. 1. If one of the mirrors is mechanically moved back and forth, the wavelength that constructively interferes at a particular incidence angle $\theta_{\mathrm{i}}$ varies, and the system acts as an interferometer. Since the transmitted angle $\theta_{\mathrm{t}}$ also depends on the refractive index $n$ of the medium between mirrors, Fabry-Pérot interferometers can also be used with a constant cavity length by changing only the optical properties of the cavity medium.

At normal incidence of light, the interferometric phase of the Fabry-Pérot interferometer is given by Eq. (2), from where it can be obtained the cavity length, $L_{\mathrm{FP}}$, as shown in Eq. (3).

$\phi=\frac{4 \pi n L}{\lambda}$

$L_{\mathrm{FP}}=\frac{\lambda^{2}}{2 n \partial \lambda}$

In this work, a FFPI sensing device with a MIP sol-gel membrane at the cleaved end of an optical fibre was used. In this case, and according to Eq. (3), $\lambda$ is the operating central wavelength at $1570 \mathrm{~nm}, n$ is the refractive index of the sensing membrane and $\partial \lambda$ is the fringe periodicity of the Fabry-Pérot channelled spectrum. The refractive index of the membrane based-sol-gel material was estimated in 1.5 , considering its constituents, and the fringe periodicity for the sensing head with the MIP membrane was approximately $44.5 \mathrm{~nm}$.

Fig. 1 shows the experimental setup used to interrogate the FFPI sensing device. It consisted of a broadband source in the $1550 \mathrm{~nm}$ spectral range with a bandwidth of $100 \mathrm{~nm}$, and an optical circulator to obtain the reflected channelled spectrum of the sensing head. The reflected Fabry-Pérot signal was observed using an optical spectrum analyser. The detail in Fig. 1 shows the developed sensing head which is based on a standard single-mode fibre tip coated with MIP or NIP sol-gel material. The fibre Fabry-Pérot cavity was then obtained by two interfering waves, one from the Fresnel reflection at the distal end of the fibre probe and the other from the reflected light at the end of the sensitive membrane deposited in the fibre tip.

\section{Experimental}

\subsection{Apparatus}

A fibre Fabry-Pérot interferometer was used. The reflected Fabry-Pérot signal was observed using an Optical Spectrum Analyser (OSA), Ando AQ-6315B, with a maximum wavelength resolution of $0.5 \mathrm{~nm}$. The fusion splicer used was a Fujikura FSM 60S, and the fibre optic stripper was a Clauss CFS-2.

For the sensing head fabrication, a standard single-mode fibre SMF-28e was used, with 8.2 and $125 \mu$ m-diameters for the core and cladding, respectively, from Corning Inc., United States. The fibre tip was cleaved with a cut machine FITEL S323 and dip-coated with a sol-gel based membrane in a home-made dip-coater. The preparation of sol-gel was done in a Yellow Line MST Basic C with temperature and stirring control. For the polimerization a Memmert TYP:U.10 was used. 

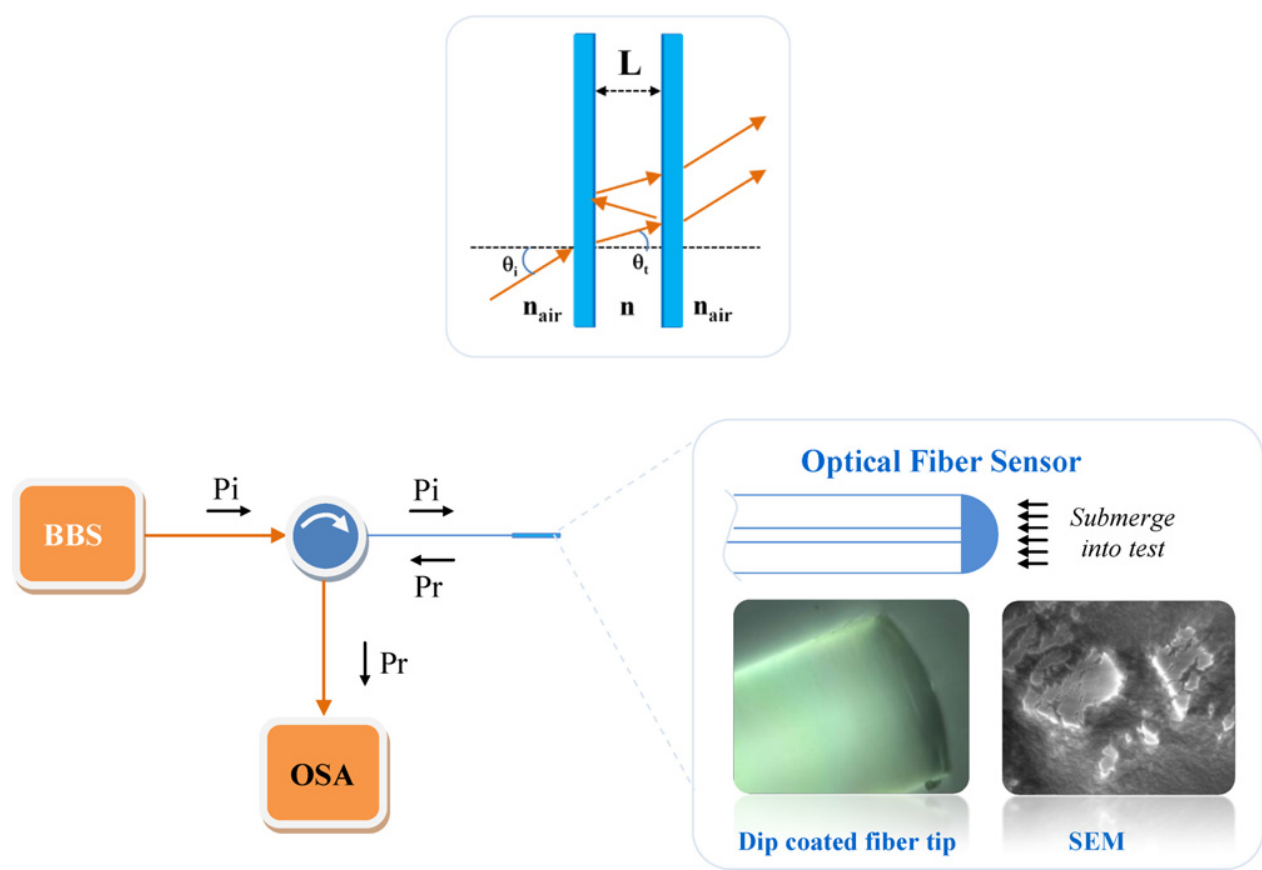


incident power; Pr: reflected power; $L$ : distance between mirrors; $\theta_{\mathrm{i}}$ : incidence angle; $\theta_{\mathrm{t}}$ : transmitted angle and $n$ : refractive index.

\subsection{Reagents and solutions}

Deionized water was used throughout. MCT (Fluka), (3-aminopropyl) trimethoxysilane (APTMS, Fluka), diphenyldimethoxysilane (DPDMS, ABCR), methanol (MeOH, Aldrich), tetraethoxysilane (TEOS, ABCR), hydrochloric acid ( $\mathrm{HCl}, \mathrm{Merck}$ ) and ethanol (EtOH, Panreac), were pro-analysis grade and used without further purification.

A stock solution of $2 \mu \mathrm{g} \mathrm{L}^{-1}$ MCT was prepared by dilution in water of $10 \mu \mathrm{L}$ of the commercial standard, a liquid methanolic solution of $10.025 \mu \mathrm{g} \mathrm{L}^{-1}$ in $50.0 \mathrm{~mL}$ of deionized water. The decrease MCT concentrations were obtained by adding aliquots of the stock solution in water till the desire concentration of MCT.

\subsection{Preparation of the MCT sol-gel membrane in a coated fibre}

The MI sol-gel membrane was applied on the cleaved end of the single-mode fibre by dip-coating. The fibre was dipped in the gel for $2 \mathrm{~min}$ at $0.483 \mathrm{~m} \mathrm{~s}^{-1}$ and then the fibre was raised at the same velocity for drying during $2 \mathrm{~min}$. This procedure was carried out 4 times.

The membrane applied was obtained by mixing $10 \mu \mathrm{L}$ of MCT with a selected sol-gel recipe composed of $3 \mathrm{~mL}$ of APTMS, $3 \mathrm{~mL}$ of DPDMS, and $10 \mathrm{~mL}$ of $\mathrm{MeOH}$. This mixture was stirred at $60^{\circ} \mathrm{C}$ for $30 \mathrm{~min}$. Then, the resulting solution was hydrolyzed slowly with $1 \mathrm{~mL}$ of TEOS, $500 \mu \mathrm{L}$ of $\mathrm{HCl} 0.1 \mathrm{M}, 5 \mathrm{~mL}$ of EtOH and $1.5 \mathrm{~mL}$ of deionised water also at $60^{\circ} \mathrm{C}$. This was made under stirring until gel formation. The fibre tip was dip coated on the mixture and polymerized at $60^{\circ} \mathrm{C}$ during $12 \mathrm{~h}$. The template was removed by washing the MIP in deionised water for $3 \mathrm{~h}$.

\subsection{Interferometric measurements}

All interferometric measurements were carried out at room temperature. The optical spectra of the FFPI of each sensor were measured in deionized water. Decreasing concentration levels of MCT were obtained by transferring aliquots of deionized water to a $10.0 \mu \mathrm{g} \mathrm{L}^{-1} \mathrm{MCT}$. The range of concentrations studied was $0.3-1.4 \mu \mathrm{g} \mathrm{L}^{-1}$.

\section{Results and discussion}

\subsection{MCT sol-gel membrane}

The process of sol-gel imprinting of MCT started by the addition of the template to the sol phase, then the mixture was hydrolysed and the matrix polymerized in a 3-D network. Finally the template was removed from the polymer, leaving vacant places behind. These places had suitable shape and functionalities for template rebinding. The NIP structured material was synthesized following the same procedure, but excluding the template from the formulation. The expected result was a similar membrane but without the cavities from MCT.

The sol-gel thin membrane was not hydrated prior to its first use. Hydration was avoided because it contributed to the instability of the analytical signal. Daily measures of the sol-gel fibre optic sensor were conducted at room temperature (unless specified otherwise) and on an acrylic homemade reactor of small capacity $(400 \mathrm{~mL})$, under continuous stirring.

The sol-gel surface looked smooth and shiny and the initial SEM images were unable to capture the underlying surface. For this reason, the material was crashed, as may be seen in Fig. 1. The subsequent SEM images were not highly resolved due to the small conductivity of the material. They confirmed the existence of nanostructures, with dimensions lying within about 40 and $80 \mathrm{~nm}$. In general, no significant differences were obtained between MIP and NIP materials.

\subsection{Optical spectra of MIP versus NIP}

The typical optical spectra of the FFPI signals obtained with MIP and NIP membranes are shown in Fig. 2. Their periodicities were $\sim 44.5 \mathrm{~nm}$ and $43.4 \mathrm{~nm}$ for the fibre tips with MIP and NIP membranes, respectively. These results indicate that in the central region 


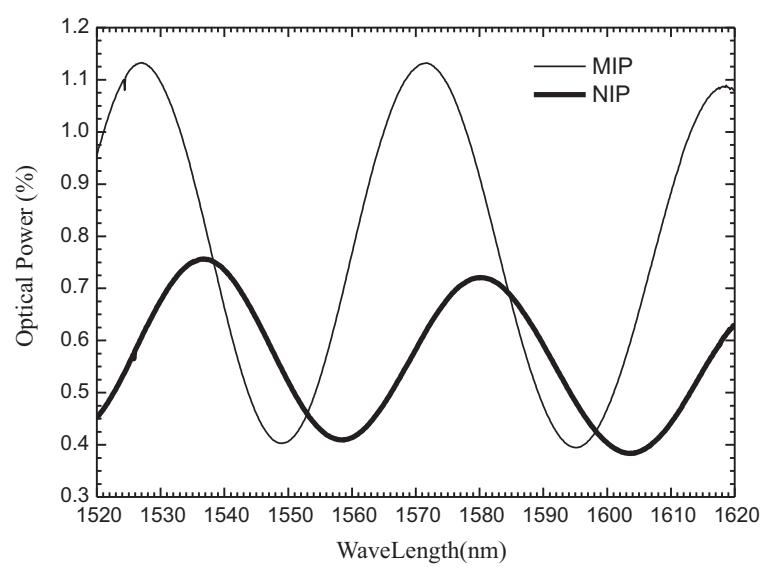

Fig. 2. Optical spectrum of the fibre for the FFPI with MIP and NIP membranes.

of the membranes the optical thicknesses were $\sim 19 \mu \mathrm{m}$. This was calculated by applying Eq. (3).

In general, the different special arrangements in MIP and NIP membranes caused different effective refractive indices which in turn were responsible by the different fringe amplitudes in the two cases. It was expectable that the cavities in the sol-gel polymer would decrease the effective refractive index of the MIP membrane, therefore causing fringes with higher amplitude when compared to the fringes of the NIP membrane due to a higher mismatch with the fibre core refractive index.

\subsection{Evidence of $M C T$ binding}

The interaction of the analyte with the sol-gel MCT membrane was evidenced by a strong influence in the phase of the FFPI. This was observed by the wavelength shift variation with the concentration of MCT. More intense shifts have been observed for imprinted sol-gel membranes. This difference was displayed for both wavelength shift and optical power, as may be seen in Figs. 3 and 4.

The great difference observed between MIP and NIP materials may result from the existence of a low number of conformational possibilities for MCT. Previous work reported that MCT in several solvents displayed only a single conformation (Trogen and Zdunek, 1996), thus contributing to the success of the molecular imprint.

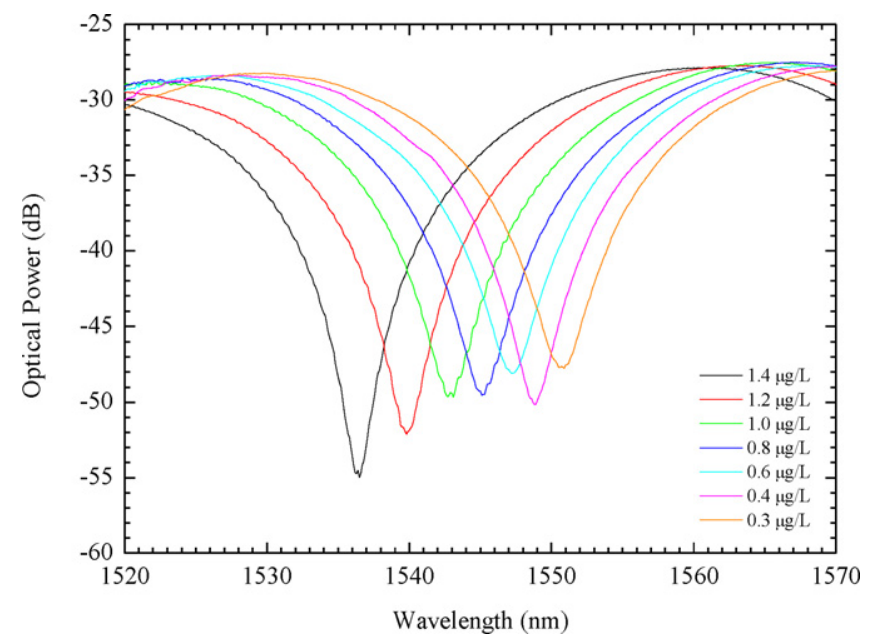

Fig. 3. Optical power and wavelength shift variation according to the concentration of MCT using an FFPI with MIP sol-gel membrane.

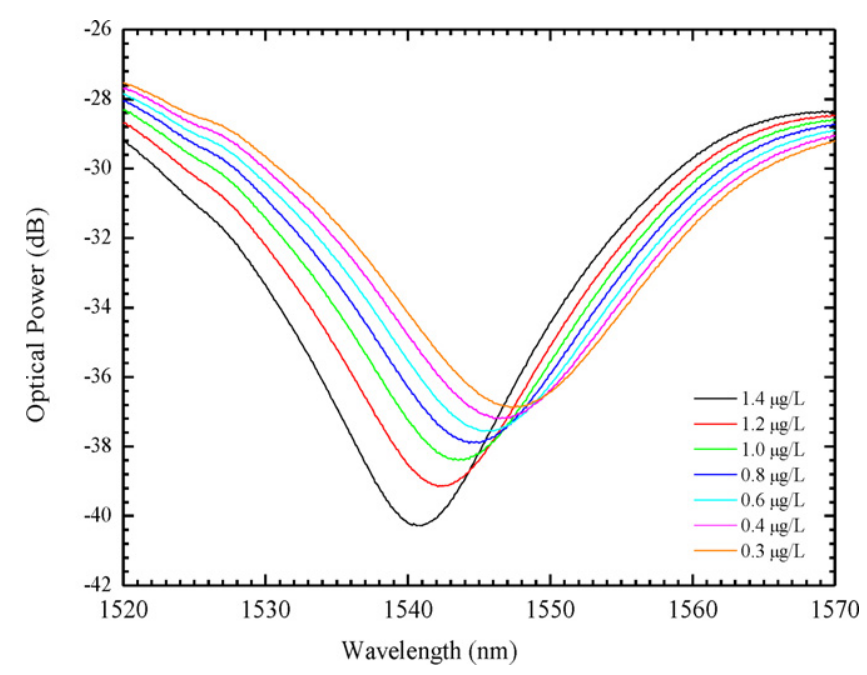

Fig. 4. Optical power and wavelength shift variation according to the concentration of MCT using an FFPI with NIP sol-gel membrane.

\subsection{Thermal-cross sensitivity}

The thermal cross-sensitivity was evaluated by immersing the sensing head in distilled water. The temperature was then increased from the room temperature up to $50^{\circ} \mathrm{C}$ with rising steps of $5^{\circ} \mathrm{C}$. The wavelength deviation was then recorded for MIP and NIP membranes.

The response of the FFPIs to temperature variation is shown in Fig. S1 in supplementary information. As it would be expected, the observed responses were non-linear. This behaviour was due to the thermo-optic coefficient variations of each sensing polymer. The FFPI with the MIP membrane displayed higher stability to temperature, which combined with its recognition binding capability indicating a great potential for MCT quantitative detection. The data presented was obtained reading with an OSA the shift of the FFPI channelled spectrum. This approach readily permitted to test the viability of this sensing structure. A dedicated interrogation unit to read with high sensitivity the cavity phase, with clear benefits considering the factors measurement resolution and cost, is now under development.

The higher thermal stability of MIP material was attributed to the presence of MCT within its matrix, as this was the only difference between NIP and MIP materials. In general terms, the interactions between the positively charged groups among MCT and the negatively charged silicate matrix (Heller and Heller, 1998) contributes to its integrity under increasing temperatures, up to $50{ }^{\circ} \mathrm{C}$. In opposite, the NIP material is expectedly more stable for higher temperatures, as any MCT molecule entrapped inside the sol-gel will be degraded. This was confirmed by differential scanning calorimetry assays indicating an endothermic alteration of MCT at $70^{\circ} \mathrm{C}$, with an enthalpy of $118.5 \mathrm{Jg}^{-1}$.

\subsection{Sensor stability}

It is a basic requirement of a chemical sensor to be able to maintain a fixed output over time when the sensor is exposed to a constant concentration of analyte (Mac-Craith et al., 1997). The perception of instability in interferometric readings with an OSA is not clear by the time of measure. The recorded signal was always a spectrum that had no perceptible correlation to the analytical signal change within time. The relative standard deviation of consecutive readings was $2 \%$, from standards ranging from 0.3 to $1.4 \mu \mathrm{gL}^{-1}$. Furthermore, the best fit for the calibration showed a correlation 
coefficient of 0.995 , indicating that the method was suitable for an analytical application.

\subsection{Response time}

The response time of sol-gel sensors is generally associated with the diffusion time of the analyte through the sol-gel matrix. Long response times are observed when electrostatic interactions are established between the matrix and charged or polar site of the analyte (Mac-Craith et al., 1997). This was the case of MCT, carrying some ionization points within its structure. Standards of higher concentration took longer time to reach a stable response, always below $2 \mathrm{~min}$. This was the maximum time taken to reach a steady wavelength of $\pm 1 \mathrm{~nm}$. Thus a period of 2 min was set for each measure. This was the time required to ensure a homogeneous background of the measuring solution and close-to equilibrium conditions in the sensing layer. In addition, using a fixed period o time avoided subsequent problems associated to the low stability of the analytical signal.

\subsection{Selectivity study}

MIPs acting as host compounds may provide a means for selectivity enhancement because the "shape" of the template is imprinted on it. The imprinted sites carry an induced molecular memory that should be capable of recognizing the previously imprinted molecules. They leave accessible binding sites with specific shape and functional group complementarily to original print molecule in the polymeric network. However, non-specific interaction is established due to the existence of opposite polarities within the template and the imprinted site. Thus, a NIP is expected to display a response for MCT, although less sensitive than that with MIP.

Considering this non-specific interaction, the interfering effect of several concomitant species was tested. According to Portuguese law (Decreto-Lei 306/2007, 2007), the most relevant ions tested on waters for human consumption are aluminum, ammonium, chloride, iron, magnesium, manganese, sodium, and sulfate. Thus the selectivity of the chemical sensor was tested against these species. Their maximum admitted levels (Table S1) were used as maximum interfering concentration because it is highly unlikely that their presence in environmental waters above these limits. Using these concentrations, the recoveries of a $1 \mu \mathrm{g} \mathrm{L}^{-1} \mathrm{MCT}$ solution ranged from 98.5 to $101.6 \%$. These values pointed out negligible interference from other species.

Some organic compounds mentioned in law were also included in this study. Benzo(a)pyrene; DCE; one pesticide, $\rho$-cyhalothrin; one PAH, benzo(b)fluoranthene; one THM compound, Chloroform; and TCE were considered for this purpose, tested at their maximum legal limits in waters. EPA 610 HAP mix was also tested to assess the effect of combined PAH's. The results, also shown in Table S1, pointed out to recoveries from 100.2 to $102.2 \%$.

\subsection{Analytical behaviour against concentration}

Two analytical features were altered by the change in concentration: the wavelength and the visibility. Figs. S2 and S3 present the wavelength variation of both FFPIs for the MCT concentration range between 0.3 and $1.4 \mu \mathrm{g} \mathrm{L}^{-1}$ at a constant temperature of $18.3 \pm 0.1^{\circ} \mathrm{C}$. The results showed that, for the full concentration range, the wavelength variation of the FFPI with MIP membrane, was $16 \mathrm{~nm}$ (Fig. S2), the double of the result found for FFPI with NIP membrane, $8 \mathrm{~nm}$ (Fig. S3). Both MIP and NIP showed a

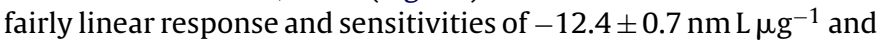

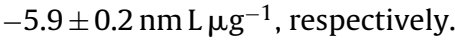

These results indicated that the presence of the analyte created a change in the effective index of the sensing membrane, introducing changes in the optical path of the cavity and, consequently, modifying the phase of the interferometric pattern. Confirming that the adsorption capacities of MIP are larger than the NIP, it was observed that MIP binding sites reached a near saturation state for higher MCT concentrations. In particular, it could be observed that, in the recommended limit range of MCT in drinking waters $\leq 1 \mu \mathrm{gL}^{-1}$, both FFPIs presented good linear behaviour with a wavelength detection range of $8 \mathrm{~nm}$ and $4 \mathrm{~nm}$ for the MIP and NIP polymers, respectively.

The dependence of the interferometric fringe visibility of both FFPIs, for the MCT concentration range between 0.3 and $1.4 \mu \mathrm{g} \mathrm{L}^{-1}$, is depicted in Figs. S4 and S5. The results showed that the fringe visibility for both FFPIs increased with the MCT concentration, meaning that this analyte changed the effective index of the sensing membranes. In the range studied, the total visibility variation was $6.6 \%$ and $4.8 \%$ for the FFPI with MIP and NIP membranes, respectively. Again, the observed differences were due to the higher adsorption capacities of the MIP membrane, since the binding sites reached faster to a near saturation state for higher MCT concentrations.

In general, the results suggest that not only the MIP but also the NIP could adsorb MCT from aqueous solutions. However, the adsorption capacities of MIP seemed much larger than those of NIP. This feature suggests that the MIP sensor may be employed nearly in the routine analysis of recreational or drinking waters. The NIP sensor capability for MCT assay in these samples, cannot however be neglected, prevented that they are applied to the analysis of real samples. This could turn out an advantage because the synthesis of the sensor is simpler and less expensive.

\subsection{Application}

In order to investigate the application of the present method to the analysis of environmental waters, spiked water solutions were tested and the corresponding relative errors calculated. These assays were conducted within $0.3-1.4 \mu \mathrm{g} \mathrm{L}^{-1}$ of MCT. The average recoveries of two spiked solutions of 1.18 and $0.77 \mu \mathrm{g} \mathrm{L}^{-1}$ were $100.4 \pm 0.6 \%$ and $96.5 \pm 2.9 \%$ and corresponding to average relative errors of $+0.57 \%$ and $-3.56 \%$ thus confirming the accuracy of the analytical data ( $n=2$ for two FFPI sensor units).

\section{Conclusions}

An interferometric optical fibre sensor coated with sol-gel MCT imprinted membranes was a successful analytical tool for screening this dangerous toxin. Both MIP and NIP sensing membranes showed linear response to $\mathrm{MCT}$ concentrations in the range of concentrations required for the analysis of recreational or drinking waters. Sensors with MIP membranes displayed higher sensitivity (double) and better detection capabilities than the corresponding NIP devices. Comparing to previous works reported in the literature for MCT determination in water, Table S2, the proposed devices are faster and less expensive. The capture-probe uses mainly synthetic materials, which accounts for the low-medium cost of the overall methodology, while alternative methods require biological materials, such as antibodies or enzymes. The developed FFPIs are promising MCT sensing units for application in situ and real time monitoring of bacteria and environment control.

\section{Acknowledgement}

RBQ thanks FCT, Fundação para a Ciência e Tecnologia, for the PhD grant SFRH/BD/49072/2008. 


\section{Appendix A. Supplementary data}

Supplementary data associated with this article can be found, in the online version, at doi:10.1016/j.bios.2011.03.015.

\section{References}

Birungi, G., Li, S.F.Y., 2009. Electrophoresis 30 (15), 2737-2742.

Bouaïcha, N., Maatouk, I., Vincent, G., Levi, Y., 2002. Food and Chemical Toxicology 40, 1677-1683.

Carmichael, W.W., 1992. Journal of Applied Bacteriology 72, 445-459.

Chianella, I., Piletsky, S.A., Tothill, I.E., Chen, B., Turner, A.P.F., 2003. Biosensors and Bioelectronics 18, 119-127.

Decreto-Lei 306/2007, 2007. Diário da República 164, 5747.

Díaz-García, M.E., Laíno, R.B., 2005. Microchimica Acta 149, 19-36.

Ding, Y., Mutharasan, R., 2011. Environmental Science and Technology 45 (4), 1490-1496.

Dunn, B., Zink, J.I., 1991. Journal of Materials Chemistry 1, 903-913.

Heller, J., Heller, A., 1998. Journal of the American Chemical Society 120, 4586-4590.

Jasionek, G., Zhdanov, A., Davenport, J.D., Blaha, L., Papkovsky, D.B., 2010. Environmental Science and Technology 44, 2535-2541.

Jesus, C., Silva, S.F.O., Castanheira, M., González-Aguilar, G., Frazão, O., Jorge, P.A.S. Baptista, J.M. 2009. Measurement Science and Technology 20 (125201), 5.

Kuiper-Goodman, T., Falconer, I.R., Fitzgerald, J., 1999. In: Chorus, I., Bartram, J. (Eds.) Human Health Aspects. E \& FN Spon, London, pp. 113-153.

Lev, O., 1992. Analysis 20, 543-553.

Li, T., Wu, T.-D., Mazéas, L., Toffin, L., Guerkin-Kern, J.-L., Leblon, G., Bouchez, T., 2008. Environmental Microbiology 10, 580-588.
Long, F., He, M., Shi, H.C., Zhu, A.N., 2008. Biosensors and Bioelectronics 23, 952-958.

Long, F., He, M., Zhu, A.N., Shi, H.C., 2009a. Biosensors and Bioelectronics 24 2346-2351.

Long, F., Shi, H.C., He, M., Sheng, J.W., Wang, J.F., 2009b. Analytica Chimica Acta 649, 123-127.

Long, F., Zhu, A., Sheng, J.-W., He, M., Shi, H.-C., 2009c. Sensors 9, 3000-3010.

Mac-Craith, B.D., Mc Donagh, C., Mcevoy, A.K., Butler, T., O’keeffe, G., Murphy, V., 1997. Journal of Sol-Gel Science and Technology 8, 1053-1061.

Mosbach, K., 1994. Trends in Biochemical Sciences 19, 9-14.

Mujahid, A., Lieberzeit, P.A., Dickert, F.L., 2010. Materials 3, 2196-2217.

Neffling, M.-R., Lance, E., Meriluoto, J., 2010. Environmental Pollution 158, 948-952.

Pyo, D., Huang, Y., Kim, Y., Hahn, J.H., 2005. Bulletin of the Korean Chemical Society 26 (6), 939.

Sangolkar, L.N., Maske, S.S., Chakrabarti, T., 2006. Water Research 40, 3485-3496.

Sheng, J., He, M., Yu, S., Shi, H., Qian, Y., 2007. Frontiers Environmental Science and Engineering China 1 (3), 329-333.

Silva, S.F.O., Frazão, O., Caldas, P., Santos, J.L., Araújo, F.M., Ferreira, L.A., 2008. Optical Engineering 47 (5), 054403.

Swanson, M.S., Hammer, B.K., 2000. Annual Review Microbiology 54, 567-613.

Triantis, T., Tsimeli, K., Kaloudis, T., Thanassoulias, N., Lytras, E., Hiskia, A., 2010. Toxicon 55, 979-989.

Trogen, G., Zdunek, J., 1996. Biochemistry 35, 3197-3205.

Vivegnis, J., El Lioui, M., Leclercq, A., Lambert, B., Decallonne, J., 1999. Biotechnology, Agronomy, Society and Environments 3, 159-164.

Wang, L., Chen, W., Xu, D., Shim, B.S., Zhu, Y., Sun, F., Liu, L., Peng, C., Jin, Z., Xu, C., Kotov, N.A., 2009. Nano Letters 9 (12), 4147-4152.

World Health Organization, 1998. Guidelines for drinking-water quality, Addendum to vol. 2., 2nd ed. Health criteria and other supporting information, Geneva. 